\title{
Management of single system prolapsed ureterocele: a case report and review of literature
}

\begin{abstract}
Background: Intralabial masses present a unique challenge for the urologist. The differential diagnosis is wide and usually outside the scope of practice for the general urologist. A prolapsed ureterocele can present as such and must be high on the differential because of the dire consequence of a missed diagnosis. Ureterocele prolapse is primarily diagnosed and treated in childhood. Despite a 1-3\% prevalence of ureteroceles in adults, prolapse among female adults is rare.
\end{abstract}

Case presentation: 54-year-old female presents to the emergency department with complaint of obstructive voiding, urine leakage, and pelvic pain. On physical exam she was found to have a $3 \times 2.5 \mathrm{~cm}$ mass protruding from her urethra with active urine leakage. She was in urinary retention with overflow incontinence. Prior imaging had diagnosed an ureterocele with associated megaureter without any evidence of renal insufficiency. The prolapsed ureterocele was reduced endoscopically into the bladder. After reduction, a $16 \mathrm{~F}$ foley catheter was placed for 7 days and removed, ensuring that she was emptying her bladder fully. At follow-up she denied any obstructive bladder emptying symptoms. She declined further surgical treatment of the ureterocele and follow-up renal Lasix scan was negative for any obstruction.

Conclusion: This case illustrates that despite the low reported incidence of prolapsed ureteroceles in adults, one must have a high index of suspicion and that conservative endoscopic treatment is an option in select patients.

Keywords: ureterocele, urinary retention, pelvic pain, intralabial mass
Volume 9 Issue 3 - 2019

\author{
Felicia Balzano, Forrest Jellison \\ Brooke Army Medical Center, USA
}

Correspondence: Forrest Jellison, Lt Col, Medical Corps, U.S. Air Force, Brooke Army Medical Center, USA, Tel 210-916-07।6 (o), Email fjellison@gmail.com

Received: May 16, 2019 | Published: May 28, 2019

\section{Introduction}

Periurethral or vulvar masses present a unique challenge for the urologist. The differential diagnosis is wide and usually outside the scope of practice for the general urologist. A prolapsed ureterocele can present as such and must be on the differential because of the consequence of a missed diagnosis. Ureterocele prolapseis primarily diagnosed and treated in childhood. Although there is a $1-3 \%$ prevalence of ureteroceles in adults, prolapse among female adults is rarely reported. The first case of single system was not reported until 2008. Since then there have been 5 other reported cases, making this a very rare condition. We aim to discuss the presentation and non-surgical endoscopic management of a single system prolapsed ureterocele and review the literature. ${ }^{1-4}$

\section{Case report}

54-year-old female presents to the emergency department with complaint of obstructive voiding, urine leakage, and pelvic pain within troital bulge. She denies any prior irritative voiding symptoms, recurrent urinary tract infections, flank pain or gross hematuria. On physical exam she was found to have a $3 \times 2.5 \mathrm{~cm}$ mass protruding from her urethra with active urine leakage from her ureteric orifice (Figure 1). Manual reduction was attempted at bedside, but was not pursued because of pain. Previously, the patient was evaluated with CT scan for microscopic hematuria, which showed a ureterocele with associated megaureter (Figure 2). Further evaluation with a voiding cystourethrogram did not reveal reflux and cystoscopy confirmed a single ureterocele without any other bladder masses (Figure 3). During this acute event of urinary retention, the patient stated that shep referred minimally invasive management, and she was taken urgently to the OR for reduction of her ureterocele under anesthesia.
Again, manual reduction was attempted, however was unable to be performed. A 22 french rigid cystoscopy was then utilized with constant, even pressure to reduce the ureterocele. After complete reduction, cystoscopy was performed demonstrating bilateral brisk efflux and a 16 french catheter was placed with return of clear yellow urine. She was discharged to home later that day with the catheter in placed. It was subsequently removed a week later and the she was able to void with a post void residual less than $50 \mathrm{ml}$. Follow up nuclear medicine functional study was performed that showed preserved renal function without evidence of obstruction. She has since been asymptomatic since reduction.

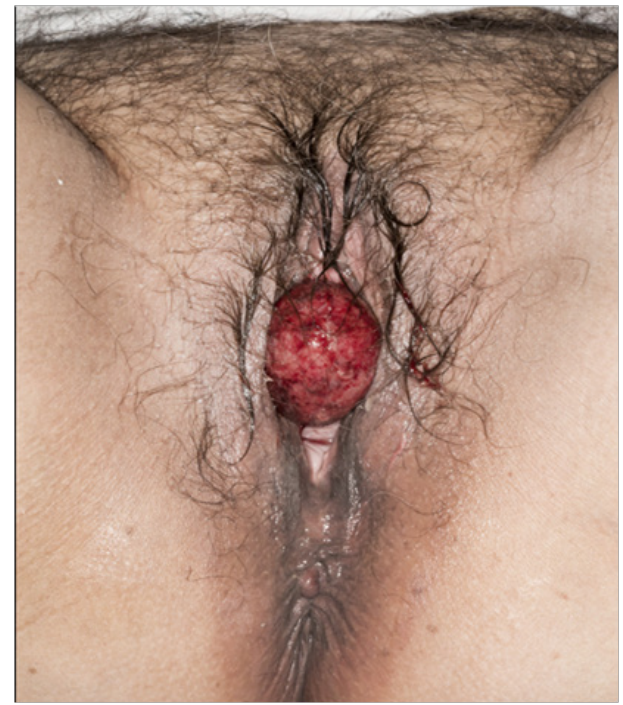

Figure I Gross picture of ureterocele prolapse. 


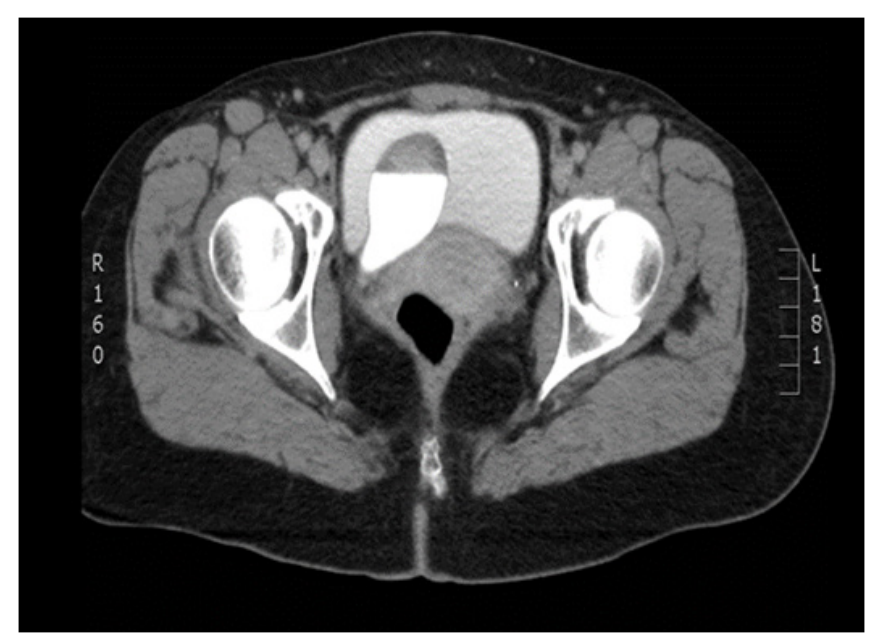

Figure 2 CT Urogram excretory phase.

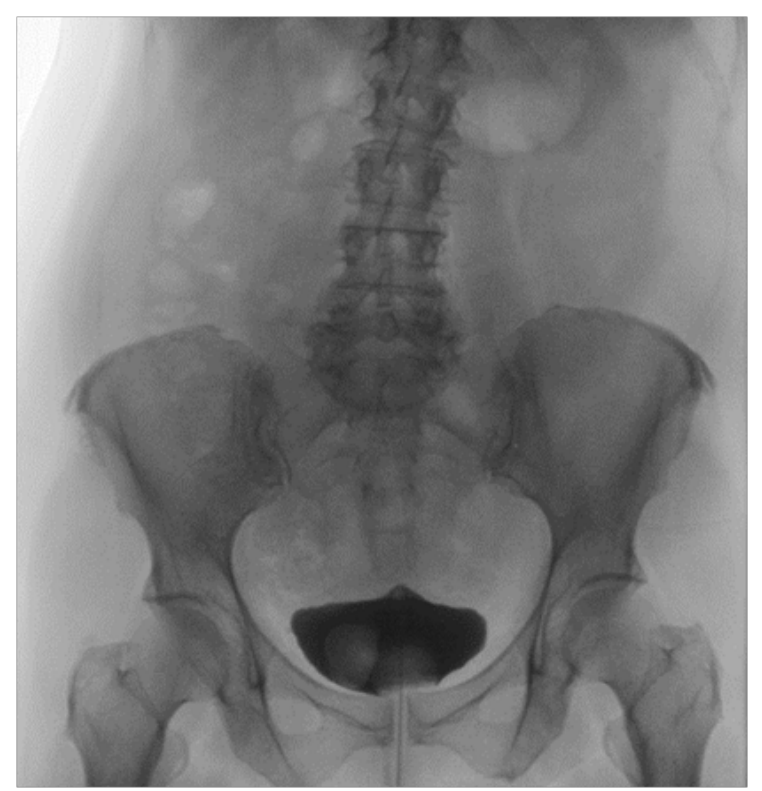

Figure 3 VCUG with ureterocele filling defect.

\section{Discussion and literature review}

Single system ureteroceles are a rare entity in the adult population, with only a $1-3 \%$ prevalence and only 8 cases have reported of prolapsed ureterocele in the literature. These can present in a wide variety of ways to include pelvic pain, urinary incontinence, urinary retention, or simply introital mass. While ureteroceles in adults usually remain asymptomatic, in the case of an ureterocele prolapse, an urologist must be involved early given the high likelihood of surgical intervention required. Manual reduction of a prolapsed ureterocele should be attempted initially because when it is successful, it can obviate a trip to the operating room. The patient should be placed in the lithotomy position. Positioning is important for these patients not only for appropriate exposure, but also to help with pelvic floor relaxation to aid in reduction.

In reviewing the literature, reports on management of single system ureterocele have been variable on patient presentation and surgeon's discretion $^{1-4}$ (Table 1). In reviewing the literature, management with reduction of the ureterocele with incision (unroofing) or reimplant were performed when it was determined there was ureteral obstruction. In our patient, manual reduction was not tolerated in the clinic so we proceeded to reduce the ureterocele in the operating room under anesthesia. Manual reduction was performed with aid of rigid scope that allowed for an even distribution of pressure to result in ureterocele reduction. After reduction has been completed, efflux of urine should be confirmed from the ureteral orifice in question. We decided to insert an indwelling catheter, although it may not have been absolutely necessary to do so. Therefore we removed it after a week and the patient was able to void freely and empty her bladder.

Table I Patient presentation of single system ureterocele prolapse.

$$
\begin{aligned}
& \text { Bleeding introital mass } \\
& \text { Vulval mass with retention } \\
& \text { Vaginal mass and incontinence } \\
& \text { Pelvic pain with urethral bulge } \\
& \text { Perineal pain and bleeding } \\
& \text { Vulval mass }
\end{aligned}
$$

Introital mass with retention and flank pain

In her follow-up after reduction she continued to be asymptomatic and functional imaging did not show any evidence of obstruction. In our opinion, patients that are conservatively managed should have follow up imaging that includes a functional study to evaluate for obstruction of the system. Discussion should be made with the patient regarding definitive management including its potential attendant complications. This would include transurethral incision (unroofing) of the ureterocele, ureteroneocystotomy or nephroureterectomy. If the patient is asymptomatic and the moiety involved does not show evidence of obstruction, it is reasonable to continue surveillance with periodic renal ultrasounds.

\section{Conclusion}

This case illustrates that despite the low reported incidence of single system prolapsed ureteroceles in adults, one must have a high index of suspicion and that conservative management is an option, although some patients will require surgical intervention.

\section{Acknowledgments}

None.

\section{Author disclosure statement}

No competing financial interests exist.

\section{References}

1. Westesson KE, Goldman HB. Prolapse of a single-system ureterocele causing urinary retention in an adult woman. Int Urogynecol J. 2013. 24(10):1761-1763.

2. Seth JH, R Walker. Case of acute severe postpartum urinary incontinence: an extravesical subsphincteric prolapsed ureterocoele. BMJ Case Rep. 2018. 2018.

3. Sinha RK, Singh S, Kumar P. Prolapsed ureterocele, with calculi within, causing urinary retention in adult female. BMJ Case Rep. 2014. 2014.

4. Villagomez-Camargo R, Chopin-Gazga M, Saucedo-Bravo J, et al. [Acute urinary retention secondary to giant prolapsed ureterocele in a young adult woman. Case report]. Cir Cir. 2016.84(4):336-339. 